\title{
Unilateral Renal Cystic Disease: A Case Report and Literature Review
}

\author{
Darmadi Darmadi ${ }^{1}$, Riska Habriel Ruslie ${ }^{2}$, Nurlaila Qodrianti Siregar ${ }^{3}$, Deli Theo ${ }^{3}$, Syahrial Anas ${ }^{3}$ \\ ${ }^{1}$ Department of Internal Medicine, Faculty of Medicine, Universitas Sumatera Utara, Medan, Indonesia; ${ }^{2}$ Department of Child \\ Health, Faculty of Medicine, Universitas Prima Indonesia, Medan, Indonesia; ${ }^{3}$ Helvetia Institute of Health, Mitra Medika Amplas \\ Hospital, Medan, Indonesia
}

Edited by: Ksenija Bogoeva-Kostovska Citation: DarD, Ruslie RH, Siregar NQ, Theo D, Anas S. Unilateral Renal Cystic Disease: A Case Report an Literature Review. Open Access Maced J Med Sci. 202 Oct 05; 8(C):160-163 https://doi.org/10.3889/oamims.2020.5031
Keywords: Cystic; Disease; Renal; Unilatera *Correspondence: Riska Habriel Ruslie, Jl. Ayahanda 68A Medan, North Sumatera, E-mail: riska habriel@yahoo com E-mail: riska.habriel@yahoo.com Revised: $17-$ Sep-2020 Accepted: $24-$ Sep-2020 Copyright: ๑ 2020 Darmadi Darmadi, Riska Habrie , Nurlaila Qodrianti Siregar, Deli Theo, Syahrial Anas Funding: This research did not receive any financial Competing Interests: The authors have declared that no competing interests exist Open Access: This is an open-accoss aticle distributed under the terms of the Creative Commons Attribution-

\begin{abstract}
BACKGROUND: URCD is a rare disease characterized by cysts with various sizes in a diffusely enlarged kidney without forming a distinct encapsulated mass. We present literature review and report a case of URCD in our center. The aim of the study was to report a case of unilateral renal cystic disease (URCD) in a 25-year-old female.

CASE REPORT: The patient was a 25-year-old female. She came to emergency unit of Mitra Medika Amplas Hospital Medan, Indonesia, with dyspepsia associated symptoms. Physical examination and family health-related history were normal. Laboratory examinations and genetic evaluation showed no abnormalities. Ultrasonography examination revealed multiple cysts in her right kidney which was confirmed by computed tomography (CT) scan The diagnosis of URCD was confirmed. No specific treatment was given and she was advised to do a routine follow-up.

CONCLUSION: URCD may present with mild symptoms or even asymptomatic. Diagnosis is confirmed by imaging modalities with normal renal function and absence of genetic predisposition. The management is conservative. Routine follow-up is mandatory.
\end{abstract}

\section{Introduction}

Unilateral renal cystic disease (URCD) is a rare disease characterized by cysts with various sizes in a diffusely enlarged kidney without forming a distinct encapsulated mass [1], [2], [3], [4], [5], [6]. Hwang et al. reported that the prevalence of URCD in their center was 2.4\% [1]. The differential diagnoses of URCD are autosomal dominant polycystic disease (ADPKD), multilocular cystic renal neoplasms, cystic dysplasia, and multiple simple cysts [1], [5], [7], [8]. It is important to distinguish URCD from ADPKD due to their different outcome [1], [3], [6], [8], [9]. URCD does not cause significant renal impairment and is not associated with genetic susceptibility [1], [2], [9], [10].

In the past, surgical modality was used to confirm URCD. Advance in imaging modality, especially computed tomography (CT) scan, may confirm the diagnosis of URCD if combined with genetic and clinical data [1], [3], [6]. The management is conservative unless clinical symptoms appear. Follow-up of renal function and imaging is mandatory [2], [7], [8]. We reported a case of URCD in a 25-year-old female and a review of literature regarding the disease.

\section{Case Report}

A 25-year-old female admitted to the emergency unit of Mitra Medika Amplas Hospital, Medan, Indonesia, with dyspepsia associated symptoms. She received a proton pump inhibitor and clinical improvement was observed. She did not experience flank pain, hematuria, fever, pain during micturition, or cloudy urine. She also denied a history of oliguria, flank pain, or hematuria. She was overall stable with a blood pressure of $120 / 80 \mathrm{mmHg}$. Physical examination showed normal results and no palpable mass at her right flank area. Both of her parents were healthy. History of renal disease and genetic abnormalities in her family were negative.

Auxiliary examination was conducted. Routine blood count was normal as well as a urinalysis. Ultrasonography examination was done and accidentally detected right kidney cysts (Figure 1). The presence of cysts was confirmed by CT scan. The presence of cysts was confirmed by CT scan (Figure 2). There were multiple cysts with various sizes in the right kidney. There was no cyst in the left kidney, liver, and pancreas. Urine albumin to creatinine ratio (UACR) was $0.25 \mathrm{mg} / \mathrm{g}$, kidney injury molecule-1 was $0.18 \mu \mathrm{g} / \mathrm{g}$, and urinary neutrophil gelatinase-associated lipocalin was 


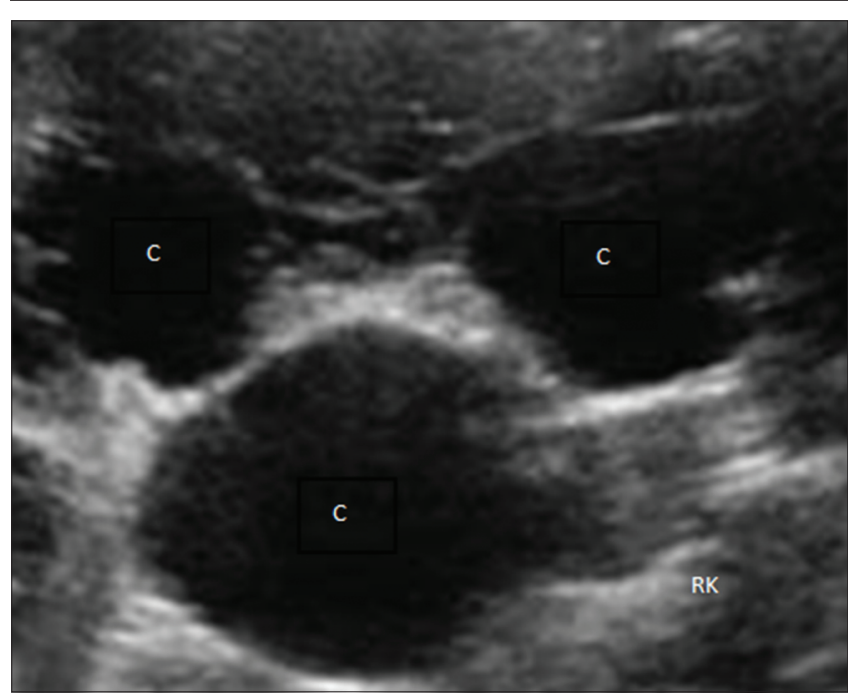

Figure 1: Cysts in the patient's right kidney observed from ultrasonography examination. RK: Right kidney, C: Cyst

$0.4 \mu \mathrm{g} / \mathrm{g}$. Serum urea level was $28 \mathrm{mg} / \mathrm{dL}$ and serum creatinine level was $0.8 \mathrm{mg} / \mathrm{dL}$. Electrolyte levels were within normal limit. Genetic evaluation was also conducted and there was no mutation in PKD1 and TCF2 genes. Her parents and siblings also underwent an abdominal ultrasonography examination and no cyst was detected. The patient refused to undergo a histopathological examination due to it is an invasive procedure.

The patient was then diagnosed with URCD with the type of multiple cysts scattered throughout the kidney separated by enhancing bands of renal parenchyma of varying width. No specific treatment was administered to the patient. She was urged to do a routine follow-up in our hospital.

\section{Discussion}

URCD was previously described as a unilateral polycystic renal disease in 1964 [2], [8]. In 1979 , several studies separated this abnormality from ADPKD [8], [11]. URCD was proposed as a distinct disease in 1989 [3], [6], [9]. Male was dominant compared to female for this disease [2], [6], [11]. Median age at diagnosis is approximately 50 years [8], [9]. Other studies reported a median age for URDC patients of 40 years [6]. The pathogenesis of URCD is unknown but suspected from a somatic mutation in the involved parenchyma [5], [9], [12]. Another hypothesis suggested an acquired maldevelopment origin as the etiology of URCD. URCD is usually diagnosed in adulthood, even though younger subjects, including neonates, may also have it [2], [8], [9]. This case was quite different from literatures above. Our case was a 25-year-old female. However, there were several reports of URCD in females at a young age.

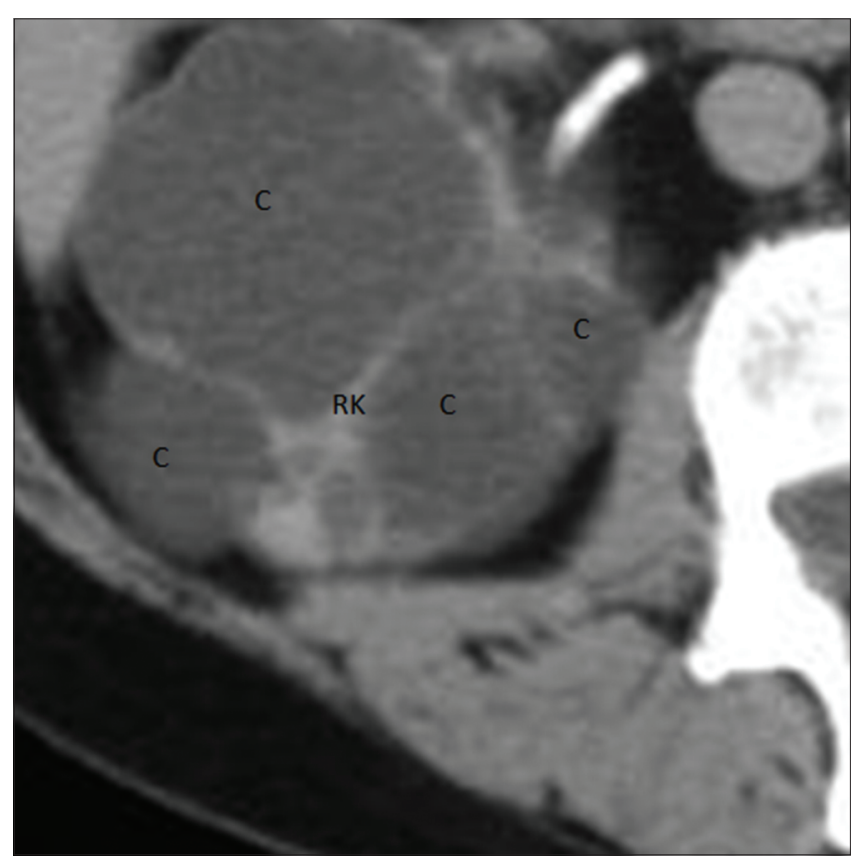

Figure 2: CT scan result showed multiple various sized cysts separated by thin renal parenchyma in the right kidney. RK: Right kidney, C: Cyst

Hwang et al. reported 2 cases of URCD in 31 and a 44-year-old male. Both had flank pain and palpable masses in the abdomen with the normal function of other organ systems. No family history with a similar complaint was found. During follow-up, both subjects did not experience renal disturbance [1]. Choh et al. also reported a male subject with URCD. He came without any symptom and URCD was suspected from routine ultrasound examination. No history of similar illness in his family and laboratory result showed normal parameters [10]. A report from Bae et al. showed a 51-year-old male with incidentally found unilateral multiple renal cysts on CT scan. His family had no renal abnormality [7]. Lee et al. reported URCD in a 72-year-old male with lower abdominal pain and mass. Serum creatinine and blood urea nitrogen levels were elevated. Other examinations were within normal limit. He had a negative family history of renal disease. CT scanning revealed multiple cystic masses in the left kidney. Histopathological examination showed similar findings with the adult polycystic disease [13].

Curry and Gordon also reported a 57-year-old male with URCD with urinary tract infection as the initial diagnosis. His urography revealed an enlarged left kidney with multiple cystic masses which confirmed by CT scan result. The right kidney was normal with normal renal function. History of family disease was negative. Follow-up showed non-progressive cysts development [4]. In another literature, a 63-year-old male came with right lumbar pain and a palpable mass at the right flank area. The presence of cysts in the right kidney was confirmed by CT scan. Histopathological examination showed a result resemble benign cyst separated by residual renal parenchyma. Other findings were unremarkable [5]. 
Baradhi and Abuelo reported a 22-year-old Asian female with URCD. Her chief complaint was abdominal pain after a motor vehicle accident. CT examination showed multiple cysts in her left kidney. No history of kidney disease in her family. Furthermore, other evaluations revealed normal results [12]. Park et al. reported URCD in a 31-year-old female. The main presentation was flank pain. Other examinations were normal and there was no history of similar familial disease. The diagnosis was confirmed by CT scan and histopathology examination [2]. An URCD case was reported by Hakkim et al. the patient was a 21-yearold female without any history of disease before. The chief complaint was flank pain. Other organ systems were normal and family history for the renal disease was negative. CT scan result showed multiple cysts in the left kidney separated by thin renal parenchyma. The treatment was conservative and the outcome was favorable [9].

A younger patient of URCD was reported by Samarkoon and Rajapakse. The case was a 17-yearold female with a chief complaint of abdominal pain. Other clinical examination and family health history were normal. CT scanning and magnetic resonance imaging showed multiple unilateral renal cysts with normal renal structure. Collecting system in URCD is not disturbed [8]. Although rare, localized cystic disease of kidney in children has been reported by Behr et al., the patient was a 6-year-old male. The chief complaint was abdominal pain. Fever and microscopic hematuria were the accompanying manifestations. Based on CT scan result, there was multiloculated cyst in the right kidney. Radical nephrectomy was conducted and histopathological examination showed various sized cysts lined by a single layer of cuboidal to hobnail epithelium and separated by thin fibrous tissue septa [11].

Maull et al. reported 3 cases of URCD. The first patient was a 32-year-old male with flank pain and gross hematuria. CT scanning showed multiple cysts in the left kidney and a single cyst in the right one. Histopathology examination showed no evidence of malignancy or dysplasia. Other findings were unremarkable. During follow-up, the cyst in the right kidney disappeared and his renal function was normal. The second patient was a 39-year-old female with a persistent cough and a lung mass. She had no urinary tract symptom or family history of renal disease. The cysts were incidentally found in the right kidney from a routine CT scan. Further follow-up showed normal renal function. The last one was a 57-year-old male with a urinary tract infection. The cysts were also incidentally found in his left kidney. No history of urinary symptom or history of renal disease was found in his family. Routine follow-up did not show the progression of renal cysts [3].

URCD is indistinguishable from ADPKD based on gross and histological findings except for its unilateral localization [1], [3], [4], [6]. URCD has five different characteristics from ADPKD: Unilateral localization, negative family history, no progression to chronic renal failure, no cysts in other intra-abdominal organs such as liver, spleen or pancreas, and no abnormality in the other organ systems [2], [3], [9], [11]. The most common symptom is flank or abdominal pain followed by palpable masses, gross or microscopic hematuria, and hypertension with normal renal function [2], [7], [8], [11]. In general, patients had a normal renal function and no history of renal associated diseases [6]. Similar to our study, the patient only had abdominal pain as a presenting symptom. Physical findings were within normal limit. Urinalysis showed normal results. Renal function test and electrolyte level were normal. History taking and auxiliary examination results from her family members were also normal.

There are two patterns of URCD: Multiple cysts scattered throughout the kidney separated by enhancing bands of renal parenchyma of varying width and cysts in one localized region of the affected kidney which are not sharply demarcated from the rest of the kidney [3], [11]. Imaging modalities, especially CT scan, hold an important role in diagnosing URCD. CT scanning result showed variablesized cysts separated by normal enhancing renal parenchyma [1], [3], [6], [9], [10]. Contrast elimination is normal in URCD which may help differentiating URCD from other conditions [11]. Genetic evaluation detecting mutations may be used to screen ADPKD and syndromes associated with renal disease [6]. The management is conservative unless clinical symptoms appeared [2], [7], [8]. In some subjects, particularly children, ADPKD may manifest as URCD. Therefore routine follow-up is important [1], [3], [9], [10]. In this report, the patient underwentabdominalultrasonography examination and the result showed multiple cysts in the right kidney. Further abdominal CT scan was utilized to confirm the abnormality. We found multiple cysts with various sizes in her right kidney and absence of cyst in other organs. The patient was diagnosed with URCD and suggested to do a routine follow-up without any medication. Genetic evaluation was also conducted to exclude renal associated syndromes and the result showed no genetic mutation in PKD1 and TCF2 genes.

\section{Conclusion}

URCD is a rare disease. It may be asymptomatic or only manifested as abdominal or flank pain. Diagnosis is made from the presence of unilateral kidney cysts with normal renal function and absence of genetic susceptibility. It is important to differentiate between URCD and other diagnoses regarding its outcome. The management is conservative and routine follow-up is mandatory. 


\section{Statement of Informed Consent}

The patient in this study had signed informed consent for being included in this report.

\section{References}

1. Hwang DY, Ahn C, Lee JG, Kim SH, Oh HY, Kim YY, et al. Unilateral renal cystic disease in adults. Nephrol Dial Transplant. 1999;14(8):1999-2003. https://doi.org/10.1093/ndt/14.8.1999 PMid:10462284

2. Park BS, Kim T, Lim S, Lee H, Jeon SH. Unilateral renal cystic disease. Korean J Urol. 2007;48(6):652-4. https://doi. org/10.4111/kju.2007.48.6.652

3. Maull CD, Curry NS, Kelty PJ, Bissada NK. Unilateral renal cystic disease. Urology. 1999;53(6):1227-8. https://doi.org/10.1016/ S0090-4295(98)00469-5

4. Curry NS, Gordon CB. Unilateral renal cystic disease in an adult. Abdom Imaging. 1994;19(4):366-8. https://doi.org/10.1007/ BF00198201

\section{PMid:8075567}

5. Punia RP, Mohan H, Bal A, Bansal VK. Unilateral and segmental cystic disease of the kidney. Int J Urol. 2005;12(3):308-10. https://doi.org/10.1111/j.1442-2042.2005.01022.x
PMid:15828961

6. Kim D, Kim M. Localized cystic disease of the kidney: CT findings. Abdom Imaging. 2003;28(4):588-92. https://doi. org/10.1007/s00261-002-0067-6

PMid:14580105

7. Bae EH, Hwang Y, Kim SW. Unilateral renal cystic disease in the right kidney. Int Braz J Urol. 2013;39(3):435-7. https://doi. org/10.1590/s1677-5538.ibju.2013.03.19

PMid:23849577

8. Samarkoon RT, Rajapakse T. Unilateral renal cystic disease: A case report of a rare disease and review of literature. Int $\mathrm{J}$ Radiol Imaging Technol. 2015;1:1.

9. Hakkim S, Babu R, Kishan RK, Mallampati SR. Unilateral renal cystic disease: A case report study. Int J Adv Med. 2019;6(2):5336. https://doi.org/10.18203/2349-3933.ijam20191171

10. Choh NA, Rashid M. Unilateral renal cystic disease. Indian J Nephrol. 2010;20(2):116-7. https://dx.doi. org/10.4103\%2F0971-4065.65310

PMid:20835331

11. Behr CA, Hesketh AJ, Williamson AK, Soffer SZ, Glick RD. Localized cystic disease of the kidney: A rare diagnosis of this uncommon condition in a child. J Pediatr Surg Case Rep. 2016;12:41-3. https://doi.org/10.1016/j.epsc.2016.06.016

12. Baradhi KM, Abuelo GJ. Unilateral renal cystic disease. Kidney Int. 2012;81:220. https://doi.org/10.1038/ki.2011.343

13. Lee JK, McClennan BL, Kissane JM. Unilateral polycystic kidney disease. AJR Am J Roentgenol. 1978;130(6):1165-7. https://doi. org/10.2214/ajr.130.6.1165

PMid:418660 